\title{
Neuroendocrine Cancer of the Breast: A Rare Entity
}

\author{
Bouhtouri Yassine ${ }^{1 *}$, Messaoudi Hamza ${ }^{1}$, Belouad Moad ${ }^{1}$, Benjilany Aboubakr ${ }^{1}$, ELouarith Ihssan $^{2}$, Moulay Abdellah \\ Babahabib $^{1}$, Mohamed Oukabli ${ }^{2}$, Kouach Jaouad ${ }^{1}$ \\ ${ }^{1}$ Department of Gynecology and Obstetrics, Military Hospital of Instruction Mohamed V Rabat, Rabat, Morocco \\ ${ }^{2}$ Department of Anatomopathology Military Hospital Mohamed V Rabat, Rabat, Morocco
}

DOI: $10.36348 /$ sijog.2021.v04i04.004

| Received: 06.03.2021 | Accepted: 07.04.2021 | Published: 11.04.2021

*Corresponding author: Bouhtouri Yassine

\section{Abstract}

Primary neuroendocrine carcinomas of the breast (NEBC) represent a rare subtype of breast cancer, accounting for 2-5\%. They generally present a more aggressive clinical form with a strong tendency to local and distant recurrence compared to other types of invasive breast carcinoma and they do not present specific clinical or radiological characteristics. We report the case study of a 58 years old female patient with primary neuroendocrine carcinoma of the breast.

Keywords: Neuroendocrine breast carcinoma, Diagnosis, Treatment.

Copyright () 2021 The Author(s): This is an open-access article distributed under the terms of the Creative Commons Attribution 4.0 International License (CC BY-NC 4.0) which permits unrestricted use, distribution, and reproduction in any medium for non-commercial use provided the original author and source are credited.

\section{INTRODUCTION}

Primary neuroendocrine carcinoma of the breast (NEBC) is a rare subtype of breast cancer [1], representing 2-5\% [2, 3] according to the World Health Organization (WHO). The incidence of NEBC remains difficult to assess since neuroendocrine markers are not routinely used in the diagnosis of breast cancer $[4,5]$.

NEBC generally present a more aggressive clinical form with a strong tendency to local and distant recurrence compared to other types of invasive breast carcinoma [6,7]. Moreover, they do not present specific clinical or radiological characteristics. The low incidence and the limited available data do not allow for optimal management of NEBC.

\section{OBSERVATION}

Patient, 58 years old, third gesture and third pare, menopausal for eight years without any hormone replacement therapy. The onset of the disease was four months before her admission with the discovery at autopalpation of a nodule in the left breast, which was progressively increasing in volume, without mastodynia or nipple discharge. The clinical examination revealed medium-sized symmetrical breasts. Examination of the left breast revealed a $4 \mathrm{~cm}$ nodule in the upper internal quadrant, poorly limited, mobile concerning both planes, without mammary discharge and inflammatory signs opposite. The contralateral breast examination was unremarkable. The lymph nodes were free. The rest of the somatic examination was normal.

Mammography revealed a $37 \times 22 \mathrm{~mm}$ left upper quadrant opacity with irregular borders classified as ACR Birads 4. The ultrasound complement revealed a hypoechoic polylobed grossly oval mass with a discrete posterior enhancement of the echoes, measuring $35 \times 24 \times 22 \mathrm{~mm}$. with an axillary adenopathy of suspicious contours.

The biopsy of the mass came back in favor of a malignant tumor proliferation with an immunohistochemical profile in favor of a small cell neuroendocrine carcinoma.

Thoraco-abdomino-pelvic scanner showed a tumor mass in the upper internal quadrant of the left breast $\left(55^{*} 45^{*} 50 \mathrm{~mm}\right)$ with subcentimetric nodes of the Berg I and II chains without any other associated lesion.

The positron emission tomography scanner showed no other suspicious focus. The treatment consisted of a left mastectomy with homolateral axillary lymph node dissection.

Histologically, the mammary tissue was a site of an invasive carcinoma formed by small cells, 
Bouhtouri Yassine et al; Sch Int J Obstet Gynec, Apr. 2021; 4(4): 99-102

arranged in solid, cordonal and trabecular patterns, with eosinophilic cytoplasm and hyperchromatic nuclei. Mitotic figures were frequent. Tumor cells were surrounded by fibrovascular stroma.Tumor was infiltrating the adipose tissue. Microscopic examination also revealed tumor emboli in vascular structures and some areas of tumor necrosis. Immunohistochemical staining showed that the cells expressed neuroendocrine markers (chromogranin, CD56). The Ki-67 cell proliferation index was approximately $90 \%$.Tumor cells were negative for estrogen and progesterone receptors. Human Epidermal Growth Factor Receptor-2 (HER2) Immunohistochemical test was negative. According to the Morphological and immunohistochemical appearances, the final diagnosis was primary small cell neuroendocrine carcinoma of the breast (figure1). The axillary lymph node curage was negative.

The adjuvant treatment consisted of six courses of ETOPOSIDE-CISPLATIN chemotherapy.

The patient received external radiotherapy at a dose of 45 grays on the chest wall, the internal mammary chain and the axillary cavity. The postoperative course was simple, with no recurrence at 12 months.

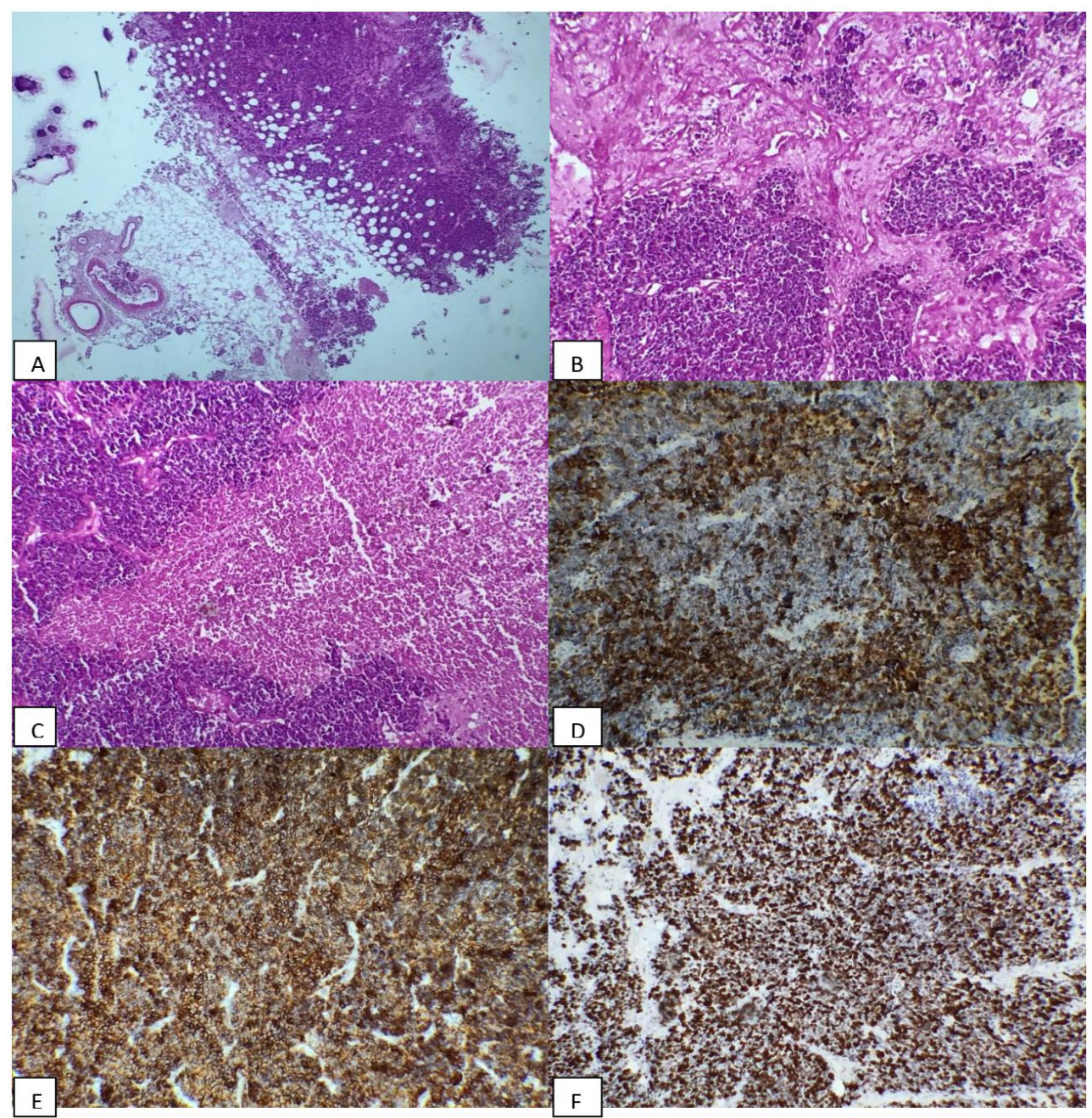

Fig-1: A 58-year-old female with left primary neuroendocrine carcinoma of the breast. A) Invasive mammary carcinoma infiltrating the adipose tissue, with vascular invasion (capillary structures are invaded by cancer cells in the peritumoral area) (hematoxylin and eosinstaining) (X10). B) Infiltrative egrowth pattern composed of small cells arranged in solid, cordonal and trabecular patterns with fibrovascular stroma (hematoxylin and eosinstaining) (X20). C) Carcinoma, solid pattern with necrosis (hematoxylin and eosinstaining) (X20). D) The tumor cells show strong expression of chromogranin (X20). E) The tumor cells show strong expression of CD56 (X20). F) Nuclear positively stained for Ki-67 estimated at $90 \%$

\section{DISCUSSION}

NEBC is defined as a carcinoma that exhibits morphologic features similar to those of neuroendocrine neoplasms of other organs. It develops forming nests and trabeculae in the fibrovascular stroma; rosettes, palisade cells and solid papillary formations can also be identified [8]. The World Health Organization (WHO) recognized NEBC as a distinct entity in 2003 [9].

Initially, WHO identified three criteria to define NEBC

1. The presence of more than $50 \%$ of neoplastic cells expressing neuroendocrine immunohistochemistry markers such as 
chromogranin A and neuron-specific synaptophysin enolase (NSE) and CD56 appear to result in lower sensitivity and specificity, mainly because they are normally present in breast tissue $[1,10,11,12]$. When neuroendocrine features are shown in less than $50 \%$ of cancer cells, the tumor should be identified as a breast cancer with neuroendocrine differentiation. Focal neuroendocrine differentiation in breast carcinoma is common and has no prognostic significance.

2. Excluding primary extra-mammary tumors, especially of the lung and gastrointestinal tract.

3. Identification of a concomitant component in situ in the breast [11].

The definition was revised in 2012, recognizing that the $50 \%$ threshold was arbitrary. Therefore, invasive carcinomas with neuroendocrine differentiation were included in the NEBC group, regardless of the percentage of tumor cells expressing neuroendocrine markers on immunohistochemistry after excluding other primary sites [1, 12]. And now distinguishes three subtypes [13]: (i) well-differentiated neuroendocrine tumors or carcinoid- like, (ii) poorly differentiated neuroendocrine tumors or small cell carcinomas, and (iii) invasive breast carcinomas with a neuroendocrine differentiation. In 2019, there was a radical change in the WHO classification of NEBC, basing on differentiation to create a common method of classification to reduce inconsistencies and contradictions between the various systems currently used.

Histologically, NEBC has a solid sheet structure of cells with a tendency to produce a peripheral palisade [14]. The tumor is often luminal subtype, positive for estrogen and progesterone receptors expression, and negative for Human Epidermal Growth Factor Receptor-2 (HER2) expression [4, 1, 7, 10, 15, 16]. Synaptophysin and chromogranin A are currently the most specific immunohistochemical markers of NEBC, whereas neuron-specific enolase and CD56 are less sensitive and specific $[5,10]$.

Clinically, mammary neuroendocrine tumors do not have specific characteristics compared to other breast tumors. They usually occur in women after the age of 60 [17]. They often present as a palpable breast mass, most frequently a single mass [18], usually larger than $2 \mathrm{~cm}$ (mean $32 \mathrm{~mm}$ in the series by Wang et al., [17], with or without axillary lymph node involvement. Rare cases of inflammatory breast cancer have been reported [10].

Radiologically, their appearance is also distinctive. On mammography, a rounded, hyperdense nodule with spiculated or micro-lobulated borders is often found [19]. On ultrasonography, a homogeneous hypoechoic solid mass with posterior enhancement is found [20], corresponding to a homogeneous hyposignal lesion that enhances early on dynamicMagnetic resonance imaging (MRI) sequences [21]. The indications for extension workup are defined by analogy with "classical" breast cancer.

The differential diagnosis of NEBC includes lymphoma, sarcoma, melanoma, and Merkel cell carcinoma and especially breast metastasis from a neuroendocrine primary of another origin [10]. The presence of an associated in situ component, hormone receptor positivity, and axillary lymph node involvement are histological arguments that point to a primary breast origin. Immunohistochemical techniques can reinforce the diagnostic hypothesis. In particular, the expression of CK7, GATA-3, GCDFP-15, mammaglobin and the absence of TTF-1 and CK20 expression by the tumor cells help to orient the pathologist towards a breast origin [22].

Prognostically, the neuroendocrine character is a pejorative factor [17]. Most authors report poorer outcomes for overall survival, local recurrence-free survival, and distant recurrence-free survival for patients with NEBC compared with the matched group of patients with invasive ductal cancer $[1,5,7,23]$.

To date, we have no prospective clinical trial data on their optimal management. It remains the same as for breast adenocarcinoma in its classic form. Surgery is the cornerstone of management. The indications for chemotherapy and radiotherapy are the same as for other breast cancers. Hormone therapy and immunotherapy have an uncertain effect; the indications are not codified [24].

\section{CONCLUSION}

NEBC is a very rare tumor associated with a more aggressive clinical course compared to other types of invasive breast cancer. The optimal treatment remains to be defined due to the limited data available. It is currently the same as conventional breast cancer. Surgery is the mainstay of treatment. Research is needed to understand the molecular profile of the tumor and to identify new targeted therapies.

Conflicts of interest: The authors declare no conflicts of interest.

\section{RÉFÉRENCES}

1. Lavigne, M., Menet, E., Tille, J. C., Lae, M., Fuhrmann, L., Bonneau, C., ... \& VincentSalomon, A. (2018). Comprehensive clinical and molecular analyses of neuroendocrine carcinomas of the breast. Modern Pathology, 31(1), 68-82.

2. Vranic, S., Palazzo, J., Sanati, S., Florento, E., Contreras, E., Xiu, J., ... \& Gatalica, Z. (2019). 
Bouhtouri Yassine et al; Sch Int J Obstet Gynec, Apr. 2021; 4(4): 99-102

Potential novel therapy targets in neuroendocrine carcinomas of the breast. Clinical breast cancer, 19(2), 131-136.

3. Cheymol, C., Abramovici, O., Do Cao, C., Dumont, A., Robin, Y. M., El Hajbi, F., ... \& Lauridant, G. (2018). Neuroendocrine tumors of the breast: Myth or reality? A systematic review. Bulletin du cancer, 105(4), 431-439.

4. Roininen, N., Takala, S., Haapasaari, K. M., Jukkola-Vuorinen, A., Mattson, J., Heikkilä, P., \& Karihtala, P. (2017). Primary neuroendocrine breast carcinomas are associated with poor local control despite favourable biological profile: a retrospective clinical study. BMC cancer, 17(1), 17.

5. Rosen, L. E., \& Gattuso, P. (2017). Neuroendocrine tumors of the breast. Archives of pathology \& laboratory medicine, 141(11), 15771581.

6. Park, Y. M., Wu, Y., Wei, W., \& Yang, W. T. (2014). Primary neuroendocrine carcinoma of the breast: clinical, imaging, and histologic features. American Journal of Roentgenology, 203(2), W221-W230.

7. Özdirik, B., Kayser, A., Ullrich, A., Savic, L. J., Reiss, M., Tacke, F., ... \& Roderburg, C. (2020). Primary neuroendocrine neoplasms of the breast: case Series and literature review. Cancers, 12(3), 733.

8. Tse, G. M., Ma, T. K., Chu, W. C., Lam, W. W., Poon, C. S., \& Wing-Cheong Chan, L. (2004). Neuroendocrine differentiation in pure type mammary mucinous carcinoma is associated with favorable histologic and immunohistochemical parameters. Modern pathology, 17(5), 568-572.

9. Hanby, A. M., Walker, C., Tavassoli, F. A., \& Devilee, P. (2004). Pathology and genetics: Tumours of the Breast and Female Genital Organs. WHO Classification of Tumours series. Vol 4. Lyon, France: IARC Press. Breast Cancer Res, 6133.

10. Inno, A., Bogina, G., Turazza, M., Bortesi, L., Duranti, S., Massocco, A., ... \& Gori, S. (2016). Neuroendocrine carcinoma of the breast: current evidence and future perspectives. The oncologist, 21(1), 28-32.

11. Abou Dalle, I., Abbas, J., Boulos, F., Salem, Z., \& Assi, H. I. (2017). Primary small cell carcinoma of the breast: a case report. Journal of medical case reports, 11(1), 1-5.

12. Talu, C. K., Leblebici, C., Ozturk, T. K., Hacihasanoglu, E., Koca, S. B., \& Gucin, Z. (2018). Primary breast carcinomas with neuroendocrine features: Clinicopathological features and analysis of tumor growth patterns in 36 cases. Annals of diagnostic pathology, 34, 122130.
13. Lakhani, S., Ellis, I., Schnitt, S., van de Vijver, M., \& Tan, P. (2012). World Health Organization classification of tumours of the breast. Lyon, France: IARC Press; p. 62-3.

14. Tavassoli, F. A., \& Devilee, P. (2003). Pathology and genetics. In: Tumors of the breast and female genital organs. WHO Classification of Tumors Series. Lyon, France, pp. 32-34.

15. Yang, X., Cao, Y., Chen, C., Liu, L., Wang, C., \& Liu, S. (2017). Primary neuroendocrine breast carcinomas: a retrospective analysis and review of literature. OncoTargets and therapy, 10, 397-407.

16. Canbak, T., Acar, A., Tolan, H. K., Ozbagriacik, M., \& Ezberci, F. Primary neuroendocrine carcinoma of the breast: a 5-year experiences. Ann Ital Chir. 91:23-26.

17. Wang, J., Wei, B., Albarracin, C. T., Hu, J., Abraham, S. C., \& Wu, Y. (2014). Invasive neuroendocrine carcinoma of the breast: a population-based study from the surveillance, epidemiology and end results (SEER) database. BMC cancer, 14(1), 1-10.

18. Rovera, F., Lavazza, M., La Rosa, S., Fachinetti, A., Chiappa, C., Marelli, M., ... \& Dionigi, R. (2013). Neuroendocrine breast cancer: retrospective analysis of 96 patients and review of literature. International Journal of Surgery, 11, S79-S83.

19. Günhan-Bilgen, I., Zekioglu, O., Ustün, E., Memis, A., \& Erhan, Y. (2003). Neuroendocrine differentiated breast carcinoma: imaging features correlated with clinical and histopathological findings. European radiology, 13(4), 788-793.

20. Yoon, Y. S., Kim, S. Y., Lee, J. H., Kim, S. Y., \& Han, S. W. (2014). Primary neuroendocrine carcinoma of the breast: radiologic and pathologic correlation. Clinical imaging, 38(5), 734-738.

21. Mariscal, A., Balliu, E., Díaz, R., Casas, J. D., \& Gallart, A. M. (2004). Primary oat cell carcinoma of the breast: imaging features. American Journal of Roentgenology, 183(4), 1169-1171.

22. Adams, R. W., Dyson, P., \& Barthelmes, L. (2014). Neuroendocrine breast tumours: breast cancer or neuroendocrine cancer presenting in the breast?. The Breast, 23(2), 120-127.

23. Wang, J., Wei, B., Albarracin, C. T., Hu, J., Abraham, S. C., \& Wu, Y. (2014). Invasive neuroendocrine carcinoma of the breast: a population-based study from the surveillance, epidemiology and end results (SEER) database. BMC cancer, 14(1), 1-10.

24. Jeon, C. H., Kim, S. M., Jang, M., Yun, B. L., Ahn, H. S., Kim, S. W., ... \& Park, S. Y. (2014). Clinical and radiologic features of neuroendocrine breast carcinomas. Journal of Ultrasound in Medicine, 33(8), 1511-1518. 